\title{
SCHINIAS WETLAND: A NATIONAL PARK OR A SOLAR SALTWORK?
}

\section{K. HADJIBIROS \\ P. SIFAKAKI}

Received: $19 / 08 / 08$

Accepted: 20/10/08

\author{
National Technical University of Athens \\ 5, Iroon Polytechniou street \\ 15780 Zografou, Athens, Greece
}

*to whom all correspondence should be addressed: e-mail: k.hadjibiros@hydro.ntua.gr

\begin{abstract}
Schinias is a small coastal biotope under environmental pressure. Partial drainage of the wetland and different disturbing activities have degraded ecological habitats and decreased biodiversity for many decades. Recently, the construction of a rowing centre has restored hydrological regime and the area has been designated as a National Park; however, application of conservation measures meets difficulties of social and financial nature. In this paper, bird diversity is used as an indicator to examine Schinias' ecosystem state, according to three different scenarios: the first one refers to the diversity that had been recorded before restoration of the hydrological regime; the second one refers to the diversity that has been recently recorded and corresponds to the actual condition of the National Park with the rowing center; the third one corresponds to a potential diversity that would be observed in case Schinias became a solar saltwork.
\end{abstract}

KEYWORDS: solar saltworks, national park, Schinias, wetland, Greece.

\section{INTRODUCTION}

Schinias is a coastal biotope in the Marathon plain, $45 \mathrm{~km}$ in the N-E of Athens, Greece. In a land area of $9 \mathrm{Km}^{2}$ there is a variety of natural habitats: freshwater spring, coastal wetland, coastal sand dunes covered by pine forest and a rocky peninsula covered by Mediterranean maquis. In the marine area there is rich posidonia vegetation. Schinias presents a particular interest as an ecosystem, as it contains more than 320 species of flora and an important diversity of animal species among which threatened resident and migrating birds, freshwater fish, amphibian and reptile species (ENVECO, 1997). Its important natural and cultural landscape is valuable for environmental education and contact with nature for four million people living in the Attica region. The state of the ecosystem depends on a fine balance between surface and underground fresh, brackish and salt water. Nevertheless, good environmental quality necessitates application of conservation measures.

Multiple and persistent anthropogenic pressures have been exerted on this area for decades. About eighty five years ago, the water of the spring has been diverted to the sea; only about $10 \%$ of water inflow remained and, therefore, the wetland has been partially drained. However, the drainage project has never been completed, only a part of the wetland has been cultivated, while the rest has remained a semi-dried marsh in which some military infrastructure and a small civil airport have been established. Pressures, like decrease of surface water and brackishness of underground water, noise and toxic pollution, solid waste disposal, uncontrolled hunting, fishing, grazing, motocross, car parking, camping and housing have resulted in considerable environmental degradation: habitat fragmentation, decrease of nesting, wintering or resident bird populations, extinction risk for endemic freshwater fish and rare plant species, no regeneration of Pinus pinea.

A rowing centre for the Olympic Games of 2004 has been planned and constructed in this area. It comprises 2 interconnected semi-natural lakes and was expected to have positive impact on the ecological functions of the biotope. The location and design principles adopted for this facility aimed at restoring the natural hydrologic regime and suppressing other disturbing uses. Water 
diverted from the spring to the sea has now been conducted to the new lakes; their overflow has been driven to the wetland. The airport and a part of military bases have been removed. An automatic forest fire suppression system feeding with water from the new lakes has been constructed. The whole area, including the rowing centre has been designated as a National Park to promote environmental protection and also recreation and sport activities (Hadjibiros, 2005).

However, realization of this ambitious integrated scheme has met difficulties, mainly of social and financial nature. The question whether a technical work can be beneficial to a biotope has received negative answers by environmental NGOs that have been strongly opposed to what they see as an artificial construction in a natural wetland; they have refused to consider the possible environmental benefits for the area from the creation of the rowing centre. Moreover, financial constraints after the Olympic Games have not allowed significant funding of the National Park; therefore additional infrastructure, e. g. construction of an external road to deviate car traffic from the National Park is still pending. On the other hand, conservation of the wetland and especially rehabilitation of its water balance, as well as implementation of measures that should restrict activities like uncontrolled car movement and parking has met opposition; an active part of the local society, especially housing and real estate associations as well as politicians of the municipality of Marathon, have continuously been trying to undermine conservation activities of the National Park. Their participation in the board of the National Park as well as efforts of Park administration to reach consensus through public hearings did not significantly change negative attitudes. Expectations that a positive change of mentalities could follow the special event of Olympic Games have not been confirmed and the question whether social conditions are favorable to the establishment of a nature conservation zone remains open. Nevertheless, there is a significant improvement of environmental conditions because of the physical changes made by the technical works.

\section{METHODOLOGY}

Nature conservation is an important driver of sustainable development. A forward-looking perspective may be essential to conserve biodiversity and safeguard natural and cultural lifesupport systems for present and future generations. Improvement of conservation tools and their dynamic adaptation to social condition is necessary. New approaches to ecological management accept that change can be a healing force in the rehabilitation of degraded areas.

Protected zones are important conservation instruments. Natural landscapes threatened by human activities should be designated as conservation areas. According to IUCN definition, National Parks are relatively large areas where ecosystems are not materially altered by human exploitation, competent authorities have taken steps to prevent degradation and visitors are allowed to enter for recreational purposes. Protection of National Parks is not efficient unless effective guarding activities take place and consensus of local societies is achieved (Jarvis, 2000).

According to Ramsar Convention, a solar saltwork is concerned to be a wetland. It may present a great interest in conserving biodiversity. Solar saltworks are series of connected ponds through which seawater flows, evaporates, and deposits salt. The ecological value of the saltworks stems from their shallow ponds that produce qualitative habitat for birds living in salt marshes and meadows, as well as for shellfish, and other animals. The biological process that develops along with the increasing salinity gradient in the evaporating ponds produces food, especially for many kinds of birds, which for this reason rest, feed and breed in the saltworks. A modern saltwork can function as an integrated saline wetland and it may reach the ecological value of a natural saline ecosystem (Den Hartog, 1981). An additional important contribution to environmental conservation is that ponds and surrounding property owned by this industrial installation are under adequate control and, therefore, provide habitat free from human disturbances for bird nesting, breeding, feeding and resting. Nevertheless, the development of a unique saline ecosystem in parallel with the salt production process has not always been understood by conservationists (Walmsley, 1999; Korovessis and Lekkas, 1999; 2006).

The environmental state of an ecosystem can be adequately assessed by use of indicators (Bosch et al., 1999). Birds are consumers that generally operate at the higher levels of trophic chains, being predators or top predators. This fact makes bird diversity an adequate indicator of the trophic functions and, therefore, of the ecosystem condition (Miller, 1996). 
In this paper, bird diversity is used as an indicator of Schinias' ecosystem state; a comparison between recorded or potential bird diversities is proposed. Three different environmental scenarios are compared: the first one refers to the bird diversity that had been recorded until 1997 and it might correspond to the "do nothing case"; the second one refers to the bird diversity that has been recently recorded and corresponds to the actual condition of the National Park with the rowing center; the third one corresponds to a potential bird diversity that would be observed in case Schinias became a solar saltwork.

\section{RESULTS}

Table 1 presents recorded or potential bird diversity corresponding to 3 different scenarios for Schinias.

\subsection{The "do nothing" scenario}

Since 1923, when the wetland was partially drained, natural habitats of Schinias have been continuously degrading. Ecological NGOs had asked for conservation measures but socioeconomic conditions in the '90s were not favoring any ambitious environmental policy. The disturbance of hydrological regime would be very difficult to mitigate, because land reclamation was a factor inhibiting any restoration of the wetland. Removal of the airport or the military base seemed extremely improbable. Implementation of restricting measures would be also difficult, because control of illegal activities required funding and support from local society. Consequently, anthropogenic pressures would probably continue or even multiply, resulting in more habitat degradation. Schinias environment would gradually become totally man-made with very low ecological value. Bird diversity recorded in the '90s (114-117 species) would not tend to increase.

\subsection{National Park with rowing centre}

In the context of the country's preparation for the Olympic Games 2004, conditions have been favorable for environmental upgrading. Following the construction of the rowing centre, Schinias has been designated as a National Park for ecology, sport and recreation. In fact, many of the expectations have not been fulfilled: the rowing centre is closed most part of the year because of financial difficulties; the new fire protection system for the forest has been abandoned and fire risk remains high; biological control of mosquitoes is still missing; funding for the Park being very low, there is no guarding; parking in the forest and on the beach, as well as other illegal activities still continue. Social support to the ambitious initial plan is too weak to face protests for wetland flooding or mosquitoes, demands to built in the wetland etc. Efficient operation of the Park as well as long term environmental protection remain uncertain. Nevertheless, four years after the Games, significant environmental improvement has been achieved: presence of a new habitat (freshwater lake); extension of freshwater marshes and vegetation; better hydrological conditions of groundwater; decrease of noise pollution and of some disturbing activities. Bird diversity goes up spectacularly: 221 species have been recorded in Schinias up to now (Stavrakas, 2008). This important increase is undoubtedly related to upgrading of natural habitats; it could also be partly attributed to more systematic observations during recent years.

\subsection{If Schinias became a solar saltwork}

A part of the Schinias wetland is a natural saline habitat. Most of the wetland area could in theory become a solar saltwork (Hadjibiros, 2006), since local climate and geomorphology are favourable for salt production. If this had happened, habitat type of salt marshes and meadows would possibly reach a surface of $6 \mathrm{~km}^{2}$. Probably, some ecological components would be subjected to a negative pressure from saltwater, for example the coastal dune forest and the freshwater habitats. Only some freshwater vegetation in the elevated part of the wetland, near the freshwater spring, could be preserved. Moreover, without the lake of the rowing centre, habitat for freshwater fish would be reduced. Persistence of the forest could be achieved, if an appropriate management of underground water was established. On the other hand, the saltwork company would be able to apply an efficient management of the wetland. It should cover the cost of conservation actions and overcome opposition from local people involved in different illegal activities. A solar saltwork is an industrial activity that cannot be impeded without cost; it may also be combined to ecotourism and develop more job opportunities; given the important economic aspect, law enforcement should be accepted and necessary restriction measures could be 
implemented in a beneficial way for wildlife. Therefore, effective protection of the biotope, and even development of ecological habitats for threatened bird species that live in Mediterranean saline wetlands would be possible. If we take account of bird diversity found in Mediterranean solar saltworks, potentially about 226 bird species could be present in Schinias (Heinzel et al., 1972; Voous, 1973; 1977; Sifakaki, 2007). Especially, bird diversity related to the habitat type "salt marshes and meadows" should reach 78 species; 7 of these have not been observed in Schinias.

Table 1. Bird diversity corresponding to the 3 scenarios for Schinias

\begin{tabular}{|c|c|c|c|}
\hline Scientific name & 을 을 & 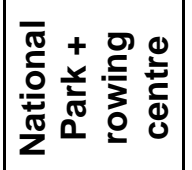 & 心 \\
\hline Garvia arctica & & & $J$ \\
\hline $\begin{array}{l}\text { Tachybaptus } \\
\text { ruficollis }\end{array}$ & $\checkmark$ & $J$ & $J$ \\
\hline Podiceps cristatus & $J$ & $J$ & $J$ \\
\hline Podiceps nigricollis & $J$ & $J$ & $J$ \\
\hline Puffinus Puffinus & & & $\sqrt{ }$ \\
\hline $\begin{array}{l}\text { Calonectris } \\
\text { diomedea }\end{array}$ & & & $J$ \\
\hline Phalacrocorax carbo & & $\checkmark$ & $\checkmark$ \\
\hline $\begin{array}{l}\text { Phalacrocorax } \\
\text { aristotelis }\end{array}$ & & $\sqrt{ }$ & $J$ \\
\hline $\begin{array}{l}\text { Phalacrocorax } \\
\text { pygmaeus }\end{array}$ & & $\checkmark$ & $\checkmark$ \\
\hline Pelecanus crispus & & & $J$ \\
\hline $\begin{array}{l}\text { Pelecanus } \\
\text { onocrotalus }\end{array}$ & & $J$ & \\
\hline Botaurus stellaris & & $J$ & $J$ \\
\hline Ixobrychus minutus & $J$ & $J$ & $J$ \\
\hline Nycticorax nycticorax & $J$ & $J$ & $J$ \\
\hline Platalea leucorodia & & & $\sqrt{ }$ \\
\hline Ardeola ralloides & $J$ & $J$ & $J$ \\
\hline Bubulcus ibis & & $J$ & \\
\hline Egretta garzetta & $J$ & $J$ & $J$ \\
\hline Egretta alba & & $J$ & $J$ \\
\hline Ardea cinerea & $\checkmark$ & $J$ & $\checkmark$ \\
\hline Ardea purpurea & $\checkmark$ & $\checkmark$ & $\checkmark$ \\
\hline Ciconia ciconia & $J$ & $J$ & $J$ \\
\hline Plegadis falcinellus & $J$ & $J$ & $J$ \\
\hline $\begin{array}{l}\text { Phoenicopterus } \\
\text { ruber }\end{array}$ & $\sqrt{ }$ & $\checkmark$ & $\checkmark$ \\
\hline Cygnus olor & $J$ & $J$ & $J$ \\
\hline Cygnus cygnus & & & $\checkmark$ \\
\hline Anser anser & & & $\checkmark$ \\
\hline Anser albifrons & & & $\sqrt{ }$ \\
\hline
\end{tabular}

\begin{tabular}{|c|c|c|c|}
\hline Scientific name & هั & 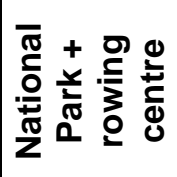 & 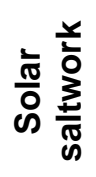 \\
\hline Branta ruficollis & & & $\checkmark$ \\
\hline Tadorna ferruginea & & $J$ & $J$ \\
\hline Tadorna tadorna & & $J$ & $J$ \\
\hline Anas penelope & & $\sqrt{ }$ & $\sqrt{ }$ \\
\hline Anas strepera & $J$ & $J$ & $J$ \\
\hline Anas crecca & & $J$ & $\bar{J}$ \\
\hline Anas platyrhynchos & $J$ & $J$ & $J$ \\
\hline Anas acuta & & $J$ & $J$ \\
\hline Anas querquedula & $J$ & $J$ & $J$ \\
\hline Anas clypeata & & $\checkmark$ & $\checkmark$ \\
\hline Netta rufina & & $\checkmark$ & \\
\hline Aythya ferina & & $\sqrt{ }$ & $J$ \\
\hline Aythya nyroca & & $\checkmark$ & $\checkmark$ \\
\hline Aythya fuligula & & $\checkmark$ & \\
\hline Bucephala clangula & & $J$ & $J$ \\
\hline Mergus serrator & & & $\checkmark$ \\
\hline Pandion haliaetus & & $\checkmark$ & $\checkmark$ \\
\hline Haliaeetus albicilla & & & $J$ \\
\hline Milvus migrans & & $J$ & \\
\hline Circaetus gallicus & & $\checkmark$ & $J$ \\
\hline Circus aeruginosus & $J$ & $J$ & $J$ \\
\hline Circus cyaneus & $J$ & $J$ & $J$ \\
\hline Circus macrourus & & $\checkmark$ & \\
\hline Circus pygargus & $\checkmark$ & $\checkmark$ & $\checkmark$ \\
\hline Accipiter gentilis & $J$ & 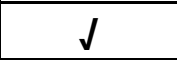 & \\
\hline Accipiter nisus & $J$ & $J$ & $J$ \\
\hline Buteo buteo & $J$ & $J$ & $J$ \\
\hline Buteo rufinus & $J$ & $J$ & $J$ \\
\hline Aquila pomarina & & $J$ & $J$ \\
\hline Aquila clanga & & $J$ & $\sqrt{ }$ \\
\hline Aquila chrysaetos & & & $\checkmark$ \\
\hline
\end{tabular}




\begin{tabular}{|c|c|c|c|c|c|c|c|}
\hline Scientific name & 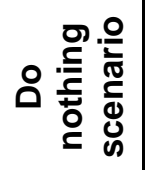 & 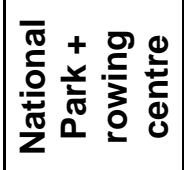 & $\frac{\frac{x}{\vdots}}{0}$ & Scientific name & هั & 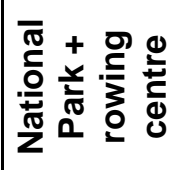 & 克 \\
\hline Hieraaetus fasciatus & & & $J$ & Calidris temminckii & $J$ & $J$ & $J$ \\
\hline Hieraaetus pennatus & & $J$ & $J$ & Calidris maritima & & & $J$ \\
\hline Gyps fulvus & & & $J$ & Calidris ferruginea & & $J$ & $\checkmark$ \\
\hline Falco naumanni & & $J$ & $J$ & Calidris alpina & & $J$ & $J$ \\
\hline Falco tinnunculus & $J$ & $J$ & $J$ & Phalaropus lobatus & & & $J$ \\
\hline Falco vespertinus & & $J$ & $J$ & Limicola falcinellus & & $J$ & $J$ \\
\hline Falco columbarius & & $J$ & $J$ & Philomachus pugnax & $J$ & $J$ & $J$ \\
\hline Falco subbuteo & & $J$ & $J$ & Gallinago gallinago & $J$ & $J$ & $J$ \\
\hline Falco biarmicus & & $J$ & J & Gallinago media & & $J$ & $J$ \\
\hline Falco peregrinus & $J$ & $J$ & J & $\begin{array}{l}\text { Lymnocryptes } \\
\text { minimus }\end{array}$ & & & $J$ \\
\hline Falco eleonorae & & & $\checkmark$ & Scolopax rusticola & $J$ & $J$ & $J$ \\
\hline Alectoris chukar & & & $\frac{1}{d}$ & Limosa limosa & J & 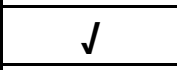 & $J$ \\
\hline$\frac{\text { Coturnix coturnix }}{\text { Rallus aquaticus }}$ & $\sqrt{l}$ & $\frac{d}{d}$ & $\frac{1}{2}$ & Limosa lapponica & & $\checkmark$ & $\checkmark$ \\
\hline Porzana porzana & $\frac{d}{d}$ & $\frac{d}{d}$ & $\frac{d}{d}$ & Numenius phaeopus & & J & $J$ \\
\hline Porzana parva & $\sqrt{ }$ & J & J & $\begin{array}{l}\text { Numenius } \\
\text { tenuirostris }\end{array}$ & & $\sqrt{ }$ & $\sqrt{ }$ \\
\hline Porzana pusilla & $J$ & $J$ & & Numenius arquata & $J$ & $J$ & $J$ \\
\hline Gallinula chloropus & $J$ & $J$ & $J$ & Tringa erythropus & 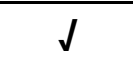 & $J$ & $J$ \\
\hline Fulica atra & $J$ & $J$ & $J$ & Tringa totanus & $\sqrt{ }$ & $J$ & $J$ \\
\hline Grus grus & $J$ & $J$ & & Thnga stagnatilis & & $J$ & $J$ \\
\hline Haematopus & & 1 & $l$ & Tringa nebularia & $J$ & $J$ & $J$ \\
\hline $\begin{array}{l}\text { Ostralegus } \\
\text { Himantopus }\end{array}$ & & & $\boldsymbol{V}$ & Thnga ochropus & $\sqrt{ }$ & $J$ & $J$ \\
\hline himantopus & J & $\checkmark$ & $J$ & Tringa glareola & $\sqrt{ }$ & $J$ & $J$ \\
\hline $\begin{array}{l}\text { Recurvirostra } \\
\text { avosetta }\end{array}$ & & & d & Xenus cinereus & & & \\
\hline Burhinus & & & & Actitis hypoleucos & & $\checkmark$ & $\checkmark$ \\
\hline oedicnemus & & & $\sqrt{ }$ & Arenaria interpres & & $\sqrt{ }$ & $J$ \\
\hline Glareola pratincola & $J$ & $J$ & $J$ & $\begin{array}{l}\text { Larus } \\
\text { melanocenhalus }\end{array}$ & J & $J$ & $J$ \\
\hline Charadrius dubius & $J$ & $J$ & $J$ & $\begin{array}{l}\text { melanocepnalus } \\
\text { Larus minutus }\end{array}$ & J & J & $J$ \\
\hline Charadrius hiaticula & & $J$ & $\checkmark$ & Larus ridibundus & J & J & $J$ \\
\hline $\begin{array}{l}\text { Charadrius } \\
\text { alexandrinus }\end{array}$ & $\checkmark$ & $\checkmark$ & $\checkmark$ & Larus genei & & $J$ & $J$ \\
\hline $\begin{array}{l}\text { Charadrius } \\
\text { leschenaultii }\end{array}$ & & & $\sqrt{ }$ & Larus audouinii & & $\checkmark$ & \\
\hline Pluvialis apricaria & & & $J$ & Larus fuscus & & $\checkmark$ & $\checkmark$ \\
\hline Pluvialis squatarola & & & $J$ & Larus michahelis & $\checkmark$ & $\checkmark$ & $\checkmark$ \\
\hline Vanellus spinosus & & $J$ & & Larus canus & & & $J$ \\
\hline Vanellus vanellus & & $J$ & $\sqrt{ }$ & Sterna nilotica & $?$ & $\checkmark$ & $J$ \\
\hline Calidris alba & & $J$ & & Sterna caspia & & & $\checkmark$ \\
\hline Calidris minutus & $J$ & $J$ & $J$ & Sterna sandvicensis & & $J$ & $J$ \\
\hline
\end{tabular}




\begin{tabular}{|c|c|c|c|}
\hline Scientific name & ○. & 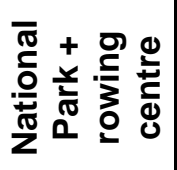 & 克 \\
\hline Sterna hirundo & & $\sqrt{ }$ & 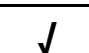 \\
\hline Sterna albifrons & & $\sqrt{ }$ & J \\
\hline Chlidonias hybridus & & $\sqrt{ }$ & J \\
\hline Chlidonias niger & & $\sqrt{ }$ & J \\
\hline $\begin{array}{l}\text { Chlidonias } \\
\text { leucopterus }\end{array}$ & & $J$ & $J$ \\
\hline Columba oenas & & J & \\
\hline Columba palumbus & & $\sqrt{ }$ & \\
\hline $\begin{array}{l}\text { Streptopelia } \\
\text { decaocto }\end{array}$ & & $\sqrt{ }$ & $\checkmark$ \\
\hline Streptopelia turtur & $\bar{J}$ & $\sqrt{J}$ & J \\
\hline Cuculus canorus & $J$ & $J$ & J \\
\hline Tyto alba & & $J$ & $J$ \\
\hline Otus scops & $J$ & $J$ & \\
\hline Bubo bubo & $?$ & $J$ & 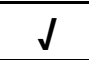 \\
\hline Athene noctua & $J$ & $J$ & $J$ \\
\hline Asio otus & J & J & \\
\hline $\begin{array}{l}\text { Caprimulgus } \\
\text { europaeus }\end{array}$ & $J$ & $\checkmark$ & $\checkmark$ \\
\hline Apus apus & 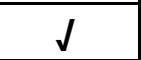 & $J$ & 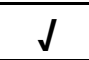 \\
\hline Apus pallidus & & $J$ & \\
\hline Apus melba & & $J$ & $J$ \\
\hline Coracias garrulus & & & J \\
\hline Alcedo atthis & $J$ & J & $J$ \\
\hline Merops apiaster & $J$ & J & $J$ \\
\hline Upupa epops & $J$ & J & $J$ \\
\hline Jynx torquilla & & 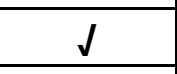 & $\checkmark$ \\
\hline Dendrocopos medius & & & $\checkmark$ \\
\hline $\begin{array}{l}\text { Calandrella } \\
\text { brachydactyla }\end{array}$ & $\checkmark$ & $\checkmark$ & $\checkmark$ \\
\hline Galerida cristata & J & J & $J$ \\
\hline $\begin{array}{l}\text { Melanocorypha } \\
\text { calandra }\end{array}$ & & & $\checkmark$ \\
\hline Lullula arborea & $J$ & $J$ & $J$ \\
\hline Alauda arvensis & $J$ & $J$ & $J$ \\
\hline Riparia riparia & & $J$ & $J$ \\
\hline $\begin{array}{l}\text { Ptyonoprogne } \\
\text { rupestris }\end{array}$ & & $\checkmark$ & \\
\hline Hirundo rustica & $J$ & J & J \\
\hline Hirundo daurica & $J$ & J & $J$ \\
\hline Delichon urbica & $J$ & $J$ & $J$ \\
\hline
\end{tabular}

\begin{tabular}{|c|c|c|c|}
\hline Scientific name & 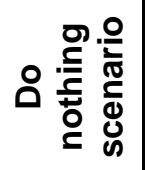 & 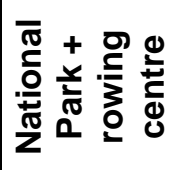 & $\frac{\frac{x}{0}}{\dot{0}}$ \\
\hline Anthus trivialis & & $J$ & $J$ \\
\hline Anthus pratensis & $J$ & $\checkmark$ & $J$ \\
\hline Anthus cervinus & & $\checkmark$ & \\
\hline Anthus campestris & & $\sqrt{ }$ & $\checkmark$ \\
\hline Anthus spinoletta & & J & $J$ \\
\hline Anthus petrosus & & J & \\
\hline Motacilla flava & J & $J$ & $J$ \\
\hline Motacilla cinerea & $J$ & $J$ & $J$ \\
\hline Motacilla alba & $J$ & J & J \\
\hline $\begin{array}{l}\text { Troglodytes } \\
\text { troglodytes }\end{array}$ & $\checkmark$ & $\checkmark$ & $\checkmark$ \\
\hline Prunella modularis & $J$ & J & J \\
\hline $\begin{array}{l}\text { Cercotrichas } \\
\text { galactotes }\end{array}$ & & $\sqrt{ }$ & $\sqrt{ }$ \\
\hline Erithacus rubecula & $J$ & J & $J$ \\
\hline $\begin{array}{l}\text { Luscinia } \\
\text { megarhynchos }\end{array}$ & $\checkmark$ & $\checkmark$ & $\checkmark$ \\
\hline $\begin{array}{l}\text { Phoenicurus } \\
\text { ochruros }\end{array}$ & & $J$ & $J$ \\
\hline $\begin{array}{l}\text { Phoenicurus } \\
\text { phoenicurus }\end{array}$ & $\checkmark$ & $J$ & $\checkmark$ \\
\hline Saxicola rubetra & $J$ & $J$ & $J$ \\
\hline Saxicola torquata & $J$ & $J$ & $J$ \\
\hline Oenanthe oenanthe & $J$ & $J$ & $J$ \\
\hline Oenanthe hispanica & & $J$ & $J$ \\
\hline Monticola solitarius & & $\checkmark$ & \\
\hline Turdus merula & $J$ & $J$ & $J$ \\
\hline Turdus pilaris & & $\checkmark$ & $J$ \\
\hline Turdus philomelos & $J$ & $J$ & $\checkmark$ \\
\hline Turdus iliacus & & $J$ & $J$ \\
\hline Turdus viscivorus & & $J$ & J \\
\hline Cettia cetti & $J$ & $\sqrt{ }$ & $J$ \\
\hline Cisticola juncidis & $J$ & $J$ & $J$ \\
\hline $\begin{array}{l}\text { Locustella } \\
\text { luscinioides }\end{array}$ & & $\checkmark$ & $\checkmark$ \\
\hline $\begin{array}{l}\text { Acrocephalus } \\
\text { melanopogon }\end{array}$ & $J$ & $\checkmark$ & $J$ \\
\hline $\begin{array}{l}\text { Acrocephalus } \\
\text { schoenobeanus }\end{array}$ & $J$ & $\checkmark$ & $J$ \\
\hline $\begin{array}{l}\text { Acrocephalus } \\
\text { scirpaceus }\end{array}$ & $J$ & $\checkmark$ & $\checkmark$ \\
\hline $\begin{array}{l}\text { Acrocephalus } \\
\text { arundinaceus }\end{array}$ & $\checkmark$ & $\sqrt{ }$ & $\sqrt{ }$ \\
\hline
\end{tabular}




\begin{tabular}{|c|c|c|c|}
\hline Scientific name & ○。 & 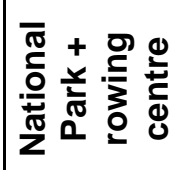 & 充 \\
\hline Hippolais pallida & $J$ & $J$ & $J$ \\
\hline Hippolais icterina & & $J$ & $\checkmark$ \\
\hline Hippolais olivetorum & & & $\checkmark$ \\
\hline Sylvia cantillans & $J$ & J & $J$ \\
\hline $\begin{array}{l}\text { Sylvia } \\
\text { melanocephala }\end{array}$ & $J$ & $J$ & $J$ \\
\hline Sylvia rueppelli & $?$ & $\checkmark$ & $\checkmark$ \\
\hline Sylvia nisoria & & & $\sqrt{ }$ \\
\hline Sylvia crassirostris & & $J$ & \\
\hline Sylvia curruca & & $J$ & $J$ \\
\hline Sylvia communis & & $J$ & $J$ \\
\hline Sylvia borin & $\bar{J}$ & $J$ & \\
\hline Sylvia atricapilla & & $J$ & $J$ \\
\hline $\begin{array}{l}\text { Phylloscopus } \\
\text { inornatus }\end{array}$ & & $J$ & \\
\hline $\begin{array}{l}\text { Phylloscopus } \\
\text { sibilatrix }\end{array}$ & & J & $\sqrt{ }$ \\
\hline $\begin{array}{l}\text { Phylloscopus } \\
\text { collybita }\end{array}$ & $J$ & $\sqrt{ }$ & $J$ \\
\hline $\begin{array}{l}\text { Phylloscopus } \\
\text { trochilus }\end{array}$ & & $\checkmark$ & $\checkmark$ \\
\hline Regulus regulus & & $J$ & $J$ \\
\hline Regulus ignicapillus & & $J$ & \\
\hline Muscicapa striata & $J$ & $J$ & $J$ \\
\hline Ficedula albicollis & & $J$ & $J$ \\
\hline Ficedula hypoleuca & & $J$ & $J$ \\
\hline Ficedula parva & & & $J$ \\
\hline Panurus biarmicus & $J$ & $J$ & \\
\hline \multicolumn{4}{|l|}{ Aegithalos caudatus } \\
\hline Parus caeruleus & & $J$ & $J$ \\
\hline Parus ater & & $J$ & \\
\hline Parus major & $J$ & $J$ & $J$ \\
\hline \multicolumn{4}{|l|}{ Parus lugubris } \\
\hline Sitta neumayer & $\checkmark$ & $J$ & \\
\hline Sitta krueperi & & & $\sqrt{ }$ \\
\hline $\begin{array}{l}\text { Certhia } \\
\text { brachydactyla }\end{array}$ & $\checkmark$ & $\checkmark$ & \\
\hline Remiz pendulinus & & $J$ & $J$ \\
\hline Oriolus oriolus & & $\checkmark$ & $\checkmark$ \\
\hline
\end{tabular}

\begin{tabular}{|c|c|c|c|}
\hline Scientific name & ه: & 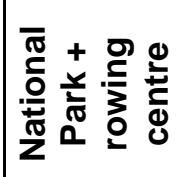 & 充 \\
\hline Lanius isabellinus & & $J$ & \\
\hline Lanius collurio & & $J$ & J \\
\hline Lanius minor & & $J$ & $J$ \\
\hline Lanius senator & $J$ & $J$ & $J$ \\
\hline Lanius nubicus & & $\sqrt{ }$ & $\sqrt{ }$ \\
\hline Lanius excubitor & & & $\checkmark$ \\
\hline Garrulus glandarius & & & $J$ \\
\hline Pica pica & $J$ & $J$ & $J$ \\
\hline Corvus cornix & $J$ & $J$ & $J$ \\
\hline Corvus corax & & $J$ & $J$ \\
\hline Corvus monedula & & & $J$ \\
\hline Corvus frugilegus & & & $J$ \\
\hline Sturnus vulgaris & $J$ & $J$ & $J$ \\
\hline Sturnus roseus & & & $J$ \\
\hline Passer domesticus & $J$ & $J$ & $J$ \\
\hline \begin{tabular}{|l} 
Passer \\
hispaniolensis
\end{tabular} & & $J$ & $J$ \\
\hline Passer montanus & $J$ & J & $J$ \\
\hline Fringilla coelebs & $J$ & $J$ & $\sqrt{ }$ \\
\hline Serinus serinus & & $J$ & $J$ \\
\hline Carduelis chloris & $J$ & $J$ & $J$ \\
\hline Carduelis carduelis & $J$ & $J$ & $J$ \\
\hline Carduelis spinus & $J$ & $J$ & \\
\hline Carduelis cannabina & $J$ & $J$ & $J$ \\
\hline $\begin{array}{l}\text { Coccothraustes } \\
\text { coccothraustes }\end{array}$ & & $J$ & \\
\hline Emberiza cirlus & $J$ & $J$ & $J$ \\
\hline $\begin{array}{l}\text { Emberiza } \\
\text { melanocephala }\end{array}$ & & $J$ & $J$ \\
\hline Emberiza cia & & $\bar{J}$ & \\
\hline Emberiza caesia & & $J$ & $J$ \\
\hline Emberiza hortulana & & & $J$ \\
\hline Emberiza rustica & & $J$ & \\
\hline \begin{tabular}{|l|} 
Emberiza \\
schoeniclus
\end{tabular} & $J$ & $\checkmark$ & $\checkmark$ \\
\hline Miliaria calandra & $J$ & $J$ & $J$ \\
\hline
\end{tabular}




\section{DISCUSSION}

Conservation measures need support from local societies. When people do not give high priority to environmental values, even valuable biotopes are subjected to degradation, unless strong external factors are involved. The "do nothing scenario" would inevitably lead to more degradation of Schinias ecological habitats.

On the other hand, if a National Park does not receive substantial financial and political support from the central government, conservation measures that do not meet aspirations of the local society are difficult to apply. In the case of Schinias, due to a major event, adequate technical works have been able to upgrade ecological functions. However, Olympic Games will not come back soon and the long-term preservation of ecological values strongly depends on uncertain socioeconomic and political conditions.

Economic activities are often useful as conservation instruments. If classical methods, like designation of a National Park, fail, alternative ways to promote coastal wetland conservation should be developed. Under certain conditions, solar saltworks may push forward environmental policy (Korovessis and Lekkas, 1999; 2006). In case a solar saltwork was developed in Schinias, bird diversity would not be less than the one corresponding in a well protected National Park in the same area; probably, 7 more bird species than those recorded in the present situation of the National Park would be expected. Moreover, an increase of populations of threatened bird species, like Falco naumanni, Falco eleonorae, Phoenicopterus rubber, Himanthopus himanthopus, Recurvirostra avosetta, Sterna albifrons, Oenanthe hispanica should be possible (Sifakaki, 2007).

Waiting for a quality artificial habitat created by a solar saltwork as well as for effective conservation measures related to an industrial activity instead of a National Park may be considered as an "ecology fiction" option. However, in some particular cases, it may also,be another way towards nature protection.

\section{ACKNOWLEDGEMENTS}

The authors would like to thank Hellenic Ornithological Society for supplying recent data on bird diversity of Schinias

\section{REFERENCES}

Bosch P., Buchele M. and Gee D., (1999) Environmental indicators: Typology and overview, European Environmental Agency.

Den-Hartog C., (1981) Aquatic plant communities of poikilo-saline waters, Hydrobiologia, 81, 1522.

ENVECO (1997). Environmental Impact Assessment of the Rowing and Kanoing Centre in Schinias-Marathon, Athens (in greek)

Hadjibiros K. (2005) The coastal Park of Schinias Marathon, In: Proc. $3^{\text {rd }}$ Conf. on "Management and Improvement of Coastal Zones", NTUA, Athens, pp. 503-516 (in greek)

Hadjibiros K., (2006) If Schinias was a saltwork? In: Proc. 1st Int. Conf. on Ecological Importance of Solar Saltworks, Santorini, 20 - 22.10.2006, pp.182-183 (ext. abstr.).

Heinzel H., Fitter R. and Parslow J. (1972) The Birds of Britain and Europe with North Africa and the Middle East, London.

Jarvis P.J. (2000) Ecological Principles and Environmental Issues, Prentice Hall

Korovessis N. and Lekkas T.D., (1999), Solar saltworks production process evolution-wetland function, In: Proc. Post-Conference Symposium "Saltworks: Preserving Saline Coastal Ecosystems, Global NEST, Samos, pp 11-30.

Korovessis N. and Lekkas T.D. (2006), Comparison of Solar Saltworks with saline coastal wetlands, In: Proc. $1^{\text {st }}$ Int. Conf. on the Ecological Importance of Solar Saltworks, Santorini, 20.10- 22.10.2006, pp.52-61.

Miller G.T., (1996), Living in the Environment: Principles, Connections and Solutions, Wadsworth Publishing Company, $9^{\text {th }}$ Ed.

Sifakaki P. (2007) Solar saltworks as productive and ecological systems: the case study of Schinias. Ms Thesis, NTUA, Athens (in greek) 
Stavrakas E. (2008) Data on bird diversity of Schinias (oral communication). Database of Hellenic Ornithological Society and Birdlife International, May 2008.

Voous K.H., (1973) List of recent holarctic bird species, Ibis, 115, 612-638.

Voous K.H., (1977) List of recent holarctic bird species, Ibis, 119, 223-250.

Walmsley J.G., (1999) The ecological Importance of Mediterranean salinas, In: Proc. PostConference Symposium "Saltworks: Preserving Saline Coastal Ecosystems, Global NEST, Samos, pp 81-95. 